\title{
Modelling and Remote Control of an Excavator
}

\author{
Yang Liu, Mohammad S Hasan, and Hongnian Yu \\ Faculty of Computing, Engineering and Technology, Staffordshire University, Stafford, UK, ST18 0AD \\ $\{$ y.liu, m.s.hasan, h.yu\}@staffs.ac.uk
}

\begin{abstract}
This paper reposts the results of an on-going project and investigates modelling and remote control issues of an industry excavator. The details of modelling, communication and control of a remotely controllable excavator are studied. The paper mainly focuses on trajectory tracking control of the excavator base and robust control of the excavator arm. These will provide the fundamental base for our next research step. In addition, extensive simulation results for trajectory tracking of the excavator base and robust control of the excavator arm are given. Finally, conclusions and further work have been identified.
\end{abstract}

Keywords - excavator; remote control; mechatronics; modelling

\section{INTRODUCTION}

Remote control of an excavator plays a significant role in real-life applications, such as nuclear decommissioning, building demolition, military operations and rescue missions, etc. The advantage of remote control is that it allows the operator to control the machine in a remote safe environment via the wired/wireless network. In order to carry out a specific task, there are two subtasks for an excavator. Firstly, the excavator has to find a feasible path from its initial location to the destination. Secondly, a robust control approach has to be designed to execute the desired excavation tasks. According to these requirements, we proposed a framework of remote control of an excavator in [1]. This paper further expands the work conducted in [1] in the following aspects: 1) modelling of the excavator base; 2) control of the excavator base; 3 ) robust control of the excavator arm; 4) remote control of the excavator base.

Based on the earlier work, implementation of a remote-control excavator mainly focused on modelling and control of the machine. The modelling work includes kinematic [2] and dynamic [3] modelling, modelling of interaction between the machine and the environment [4] and parameter identification [5][6][7]. The key reason for modelling and parameter identification during the digging operation is to provide online parameters for the real-time monitoring and remote control. In [5], a novel approach for experimental determination of the joint parameters and friction coefficients was developed on the excavator arm. Zweiri et al. [6] presented another robust, fast, and simple technique for the experimental identification of the joint parameters and friction coefficients of a full-scale excavator arm. Furthermore, an online soil parameter estimation scheme was proposed in [7]. During the earlier stage of excavation control, impedance control was considered as a prevalent robust control approach to achieve compliant motion in contact tasks. In [8], a position-based impedance controller was presented on various contact experiments by using an instrumented mini-excavator. Details of robust impedance control for a hydraulic excavator have been presented in [9][10].

In contrast to control of the excavator arm, motion control and path planning for the excavator base have also been studied in a number of research papers [11][12]. In [13], a vision-based control system for a tracked excavator was presented. The system includes several controllers that collaborate to move the excavator from a starting position to a goal position. Furthermore, a number of researchers have investigated the feasibility of remotecontrol excavation. Many of these studies have addressed the possible use of the remote control approach on the excavator [1][8]. However, in a remote-control excavator system, if the operator cannot sense the condition of contact, the work efficiency will decrease in comparison to direct control by the human operator. So, design of the joystick with proper force feedback [14][15] is key to controlling an excavator remotely. The joystick can make skilful operators adapt their operation to the excavating environment based on their empirical knowledge, and can further realize efficient excavation. Moreover, in contrast to controlling a real hydraulic excavator, there are many studies which implement their work on the virtual excavator [16][17]. The virtual excavator system appears to be a low-cost, safe, and reliable system that can be used to test both the system and the control strategy in a virtual environment.

As discussed above, many research studies have focused on the modelling and controller development stages, but there is less literature studying the remote operation from a network communication point of view. Furthermore, it is found that efficiency of excavation by a human operator [18] is a notable issue that has potential commercial value. On the other hand, a remote-control excavator has been the wish of both industry and manufacturing over the past two decades. Much of the work on terrestrial excavation has focused on teleoperation, rather than on the system requirements for autonomous operation. However, although remarkable and valuable progress has been made on automated excavation, remote control of a full-scale excavator has not been commercially demonstrated.

In this paper, we will report the further development based on the work in [1] and will focus on the work from the following two aspects: 1) trajectory tracking control of the excavator base and 2) robust control of the excavator arm. Section II studies the models of the excavator base and the excavator arm. Those models will provide the basis for the system design, development of the controllers, task/path planning, simulation, and validation, etc. Section III investigates the control approaches for controlling the excavator base and the excavator arm. Section IV proposes a wireless networked control scheme 
for the excavator base. Finally, the conclusions and future work are given in section $\mathrm{V}$.

\section{MODELLING OF AN EXCAVATOR}

\section{A. Modelling of the excavator base}

The excavator base model is taken from [19] and [20]. It has two driving wheels and a free rotating front wheel. Two wheels are independently driven by actuators to produce transition and orientation. The excavator base model is shown in Figure 1, and the parameters are given in Appendix. The centre of mass and the centre of the excavator base gear are represented by points $\mathrm{C}$ and $\mathrm{A}$, respectively.

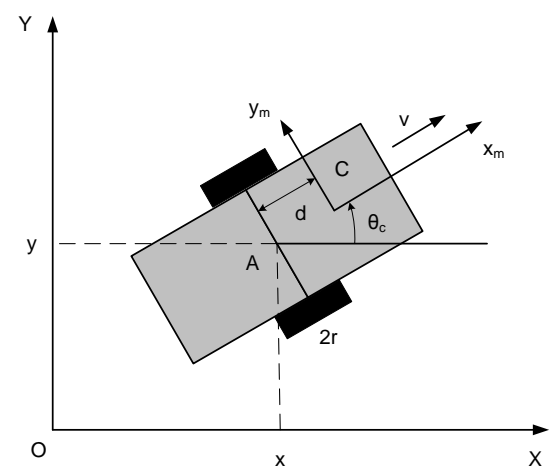

Figure 1 The excavator base model for position control given in [19] and [20].

The kinematic model of the excavator base is presented as below.

$$
\dot{x}_{A}=\frac{r}{2}\left(\dot{\theta}_{R}+\dot{\theta}_{L}\right) \cos \theta
$$

$$
\begin{aligned}
\dot{y}_{A} & =\frac{r}{2}\left(\dot{\theta}_{R}+\dot{\theta}_{L}\right) \sin \theta \\
\dot{\theta}_{c} & =r\left(\dot{\theta}_{R}-\dot{\theta}_{L}\right) / 2 R
\end{aligned}
$$

The dynamic model of the excavator base is expressed as follows.

$$
D_{c} \ddot{q}+C_{c} \dot{q}=\tau_{c}
$$

where

$q=\left[\begin{array}{l}\theta_{R} \\ \theta_{L}\end{array}\right], D_{c}=\left[\begin{array}{ll}A & B \\ B & A\end{array}\right], C_{c}=\left[\begin{array}{cc}0 & K \\ K & 0\end{array}\right], \tau_{c}=\left[\begin{array}{l}\tau_{R} \\ \tau_{L}\end{array}\right]$

$A=\frac{M r^{2}}{4}+\frac{\left(I_{A}+M d^{2}\right) r^{2}}{4 R^{2}}+I_{0}$

$B=\frac{M r^{2}}{4}-\frac{\left(I_{A}+M d^{2}\right) r^{2}}{4 R^{2}}$

and $K=A / 2$.

\section{B. Modelling of the excavator arm}

The dynamic model of the excavator can be expressed concisely using the form of the well-known rigid-link manipulator equations of motion [21]:

$$
D_{a}(\theta) \ddot{\theta}+C_{a}(\theta, \dot{\theta}) \dot{\theta}+G_{a}(\theta)+B_{a}(\dot{\theta})=\Gamma \tau_{a}-F_{L}
$$

where $\theta=\left[\begin{array}{llll}\theta_{1} & \theta_{2} & \theta_{3} & \theta_{4}\end{array}\right]^{T}$ is the vector of measured joint angles as defined in Figure 2; $D_{\mathrm{a}}(\theta)$ represents inertia; $C_{a}(\theta, \dot{\theta})$ represents Coriolis and centripetal effects; $\mathrm{G}_{\mathrm{a}}(\theta)$ represents gravity forces; $B_{a}(\dot{\theta})$ represents frictions; $\Gamma$ is the corresponding input matrix; vector $\tau_{\mathrm{a}}=\left[\begin{array}{llll}\tau_{1} & \tau_{2} & \tau_{3} & \tau_{4}\end{array}\right]^{\mathrm{T}}$ specifies the torques acting on the joint shafts; $F_{L}$ represents the interactive torques between the bucket and the environment during the digging operation.

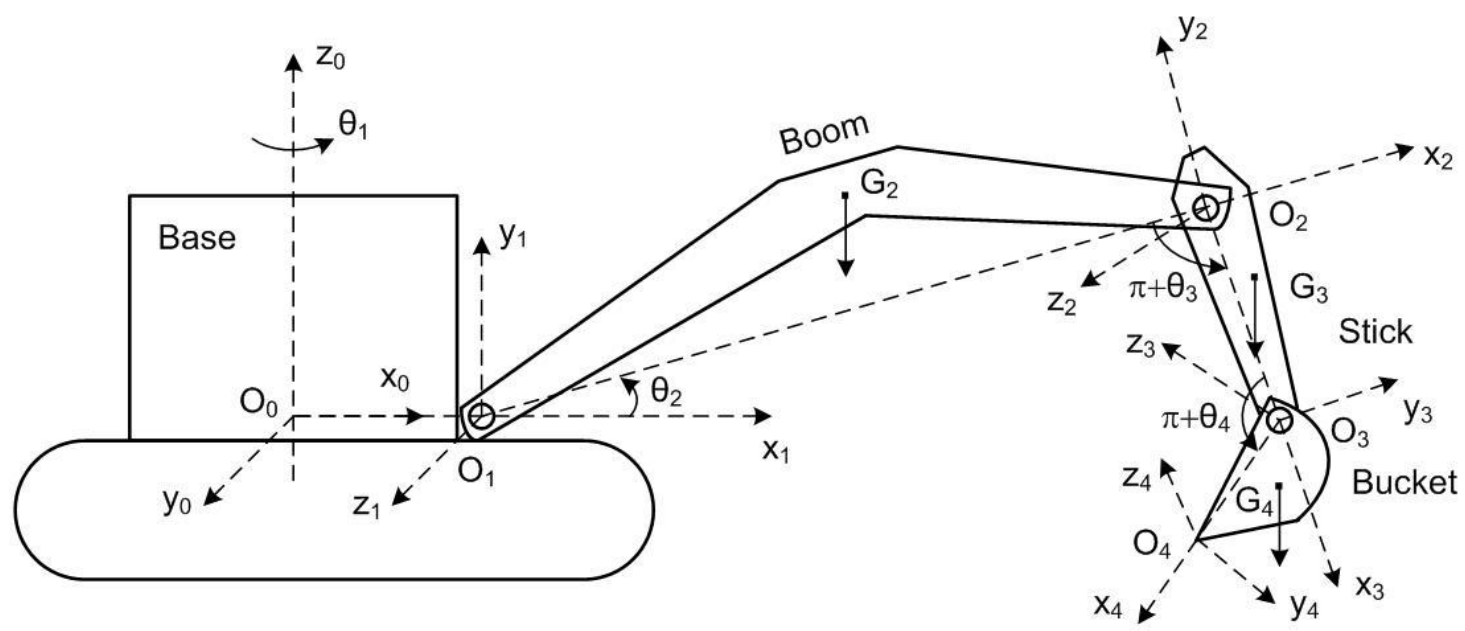

Figure 2 Schematic diagram of an excavator [4]

According to [4], $\mathrm{D}_{\mathrm{a}}(\theta), C_{a}(\theta, \dot{\theta}), \mathrm{G}_{\mathrm{a}}(\theta), \Gamma$, and $\mathrm{F}_{\mathrm{L}}$ are given by the following expression:

$$
D_{a}(\theta)=\left[\begin{array}{llll}
D_{11} & D_{12} & D_{13} & D_{14} \\
D_{21} & D_{22} & D_{23} & D_{24} \\
D_{31} & D_{32} & D_{33} & D_{34} \\
D_{41} & D_{42} & D_{43} & D_{44}
\end{array}\right]
$$

$$
\begin{aligned}
D_{44} & =I_{b u}+M_{b u} r_{4}^{2} \\
D_{33} & =D_{44}+I_{s t}+M_{s t} r_{3}^{2}+M_{b u}\left[a_{3}^{2}+2 a_{3} r_{4} \cos \left(\theta_{4}+\alpha_{4}\right)\right] \\
D_{22} & =D_{33}+I_{b o}+M_{b o} r_{2}^{2}+M_{s t}\left[a_{2}^{2}+2 a_{2} r_{3} \cos \left(\theta_{3}+\alpha_{3}\right)\right] \\
& +M_{b u}\left[a_{2}^{2}+2 a_{2} a_{3} c_{3}+2 a_{2} r_{4} \cos \left(\theta_{34}+\alpha_{4}\right)\right] \\
D_{34} & =D_{43}=D_{44}+M_{b u} a_{3} r_{4} \cos \left(\theta_{4}+\alpha_{4}\right) \\
D_{24} & =D_{42}=D_{34}+M_{b u} a_{2} r_{4} \cos \left(\theta_{34}+\alpha_{4}\right)
\end{aligned}
$$

where 


$$
\begin{gathered}
D_{23}=D_{32}=D_{24}+I_{s t}+M_{s t}\left[r_{3}^{2}+a_{2} r_{3} \cos \left(\theta_{3}+\alpha_{3}\right)\right] \\
+M_{b u}\left[a_{3}^{2}+a_{2} a_{3} c_{3}+a_{3} r_{4} \cos \left(\theta_{4}+\alpha_{4}\right)\right] \\
C_{a}(\theta, \dot{\theta})=\left[\begin{array}{llll}
C_{11} & C_{12} & C_{13} & C_{14} \\
C_{21} & C_{22} & C_{23} & C_{24} \\
C_{31} & C_{32} & C_{33} & C_{34} \\
C_{41} & C_{42} & C_{43} & C_{44}
\end{array}\right]
\end{gathered}
$$

where

$$
\begin{aligned}
& C_{22}=-M_{s t} a_{2} r_{3} \dot{\theta}_{23} \sin \left(\theta_{3}+\alpha_{3}\right)-M_{b u} a_{2} a_{3} \dot{\theta}_{23} s_{3} \\
&-M_{b u} a_{2} r_{4} \dot{\theta}_{234} \sin \left(\theta_{34}+\alpha_{4}\right) \\
& C_{23}=-M_{s t} a_{2} r_{3} \dot{\theta}_{23} \sin \left(\theta_{3}+\alpha_{3}\right)-M_{b u} a_{2} a_{3} \dot{\theta}_{23} s_{3} \\
&-M_{b u} a_{2} r_{4} \dot{\theta}_{234} \sin \left(\theta_{34}+\alpha_{4}\right) \\
& C_{24}=-M_{b u} a_{2} r_{4} \dot{\theta}_{234} \sin \left(\theta_{34}+\alpha_{4}\right) \\
& C_{32}= a_{2} \dot{\theta}_{2}\left[M_{b u} a_{3} s_{3}+M_{s t} r_{3} \sin \left(\theta_{3}+\alpha_{3}\right)\right] \\
&-M_{b u} a_{3} r_{4} \dot{\theta}_{234} \sin \left(\theta_{4}+\alpha_{4}\right) \\
& C_{33}=-M_{b u} a_{3} r_{4} \dot{\theta}_{234} \sin \left(\theta_{4}+\alpha_{4}\right) \\
& C_{34}=-M_{b u} a_{3} r_{4} \dot{\theta}_{234} \sin \left(\theta_{4}+\alpha_{4}\right) \\
& C_{42}= M_{b u} r_{4} \dot{\theta}_{2}\left[a_{2} \sin \left(\theta_{34}+\alpha_{4}\right)+a_{3} \sin \left(\theta_{4}+\alpha_{4}\right)\right] \\
&+M_{b u} a_{3} r_{4} \dot{\theta}_{3} \sin \left(\theta_{4}+\alpha_{4}\right) \\
& C_{43}= M_{b u} a_{3} r_{4} \sin \left(\theta_{4}+\alpha_{4}\right) \\
& C_{44}= 0 \\
& G_{a}(\theta)=\left[\begin{array}{llll}
G_{1} & G_{2} & G_{3} & G_{4}
\end{array}\right]^{T}
\end{aligned}
$$

where

$$
\begin{array}{r}
G_{2}=\left(M_{b u}+M_{s t}\right) g a_{2} c_{2}+M_{b o} g r_{2} \cos \left(\theta_{2}+\alpha_{2}\right) \\
G_{3}=M_{b u} g a_{3} c_{23}+M_{s t} g r_{3} \cos \left(\theta_{23}+\alpha_{3}\right) \\
G_{4}=M_{b u} g r_{4} \cos \left(\theta_{234}+\alpha_{4}\right) \\
B_{a}(\dot{\theta})=\left[\begin{array}{llll}
B_{b a} \dot{\theta}_{1} & B_{b o} \dot{\theta}_{2} & B_{s t} \dot{\theta}_{3} & B_{b u} \dot{\theta}_{4}
\end{array}\right]^{T} \\
\Gamma=\left[\begin{array}{cccc}
\Gamma_{11} & \Gamma_{12} & \Gamma_{13} & \Gamma_{14} \\
\Gamma_{21} & 1 & -1 & 0 \\
\Gamma_{31} & 0 & 1 & -1 \\
\Gamma_{41} & 0 & 0 & 1
\end{array}\right]
\end{array}
$$

The interaction between the excavator bucket and the environment is presented in Figure 3.

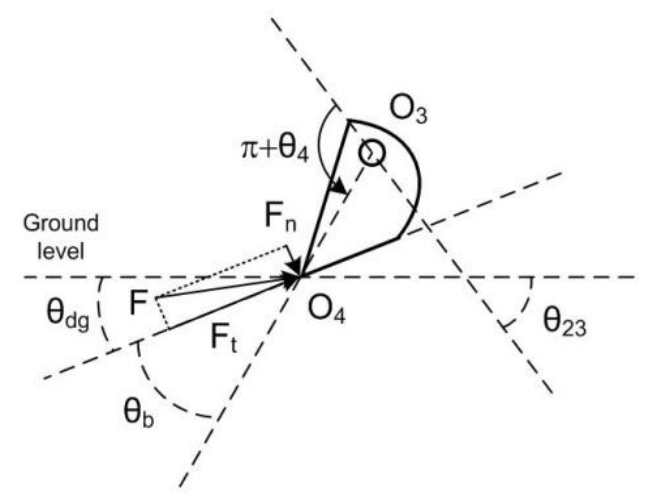

Figure 3 The interaction between the excavator bucket and the environment [4]

According to [22], $F_{t}$ and $F_{n}$ are the tangential and normal components of the soil reaction force at the bucket, respectively. The tangential component can be calculated as

$$
F_{t}=k_{1} b h
$$

where $\mathrm{k}_{1}$ is the specific digging force in $\mathrm{N} / \mathrm{m}^{2}$, and $\mathrm{h}$ and $\mathrm{b}$ are the thickness and width of the cut slice of soil. The normal component $\mathrm{F}_{\mathrm{n}}$ is calculated as

$$
F_{n}=\Psi F_{t}
$$

where $\Psi=0.1-0.45$ is a dimensionless factor that depends on the digging angle, digging conditions, and the wear of the cutting edge.

So according to Figure 3, the loading torque is given as below:

$$
F_{L}=\left[\begin{array}{c}
\tau_{b} \\
a_{2}\left[F_{t} \sin \left(\theta_{2}-\theta_{d g}\right)-F_{n} \cos \left(\theta_{2}-\theta_{d g}\right)\right] \\
a_{3}\left[F_{t} \sin \left(\theta_{23}+\theta_{d g}\right)-F_{n} \cos \left(\theta_{23}+\theta_{d g}\right)\right] \\
-a_{4}\left(F_{t} \sin \theta_{b}+F_{n} \cos \theta_{b}\right)
\end{array}\right]
$$

Since this paper is mainly on the motion control, the elements $\mathrm{D}_{1 \mathrm{i}}, \mathrm{D}_{\mathrm{i} 1}, \mathrm{C}_{1 \mathrm{i}}, \mathrm{C}_{\mathrm{i} 1}, \Gamma_{1 \mathrm{i}}, \Gamma_{\mathrm{i} 1}\left(\mathrm{i}=1,2,3\right.$, and 4), $\mathrm{G}_{1}$, $\mathrm{B}_{\mathrm{ba}}, \tau_{1}$ and $\tau_{\mathrm{b}}$ are not used in the proposed control law. However, those parameters are important in the forced control and will be investigated in the future work.

\section{CONTROL OF AN EXCAVATOR}

\section{A. Control of the excavator base}

Two PD controllers have been implemented and tuned as suggested in [19] and [20]. Equations (14) to (19) are applied to control the right and left wheel actuator torques. The gains of the applied PD controllers are given in Table 1.

$$
\begin{gathered}
e_{v}(t)=v_{r e f}(t)-v(t)=u_{1}-v(t) \\
e_{\omega}(t)=\omega_{r e f}(t)-\omega(t)=u_{2}-\omega(t) \\
u_{v}(t)=K_{p v} e_{v}(t)+K_{i v} \int e_{v}(t) d(t) \\
u_{\omega}(t)=K_{p \omega} e_{\omega}(t)+K_{i \omega} \int e_{\omega}(t) d(t) \\
u_{R}(t)=\tau_{R}=\frac{1}{2} u_{v}(t)+\frac{1}{2} u_{\omega}(t) \\
u_{L}(t)=\tau_{L}=\frac{1}{2} u_{v}(t)-\frac{1}{2} u_{\omega}(t)
\end{gathered}
$$

\begin{tabular}{|l|l|l|}
\hline Parameter & Description & Value \\
\hline $\mathrm{K}_{\mathrm{pv}}$ & $\begin{array}{l}\text { The proportional component for the forward } \\
\text { speed control }\end{array}$ & 6.48 \\
\hline $\mathrm{K}_{\mathrm{iv}}$ & $\begin{array}{l}\text { The integral component for the forward speed } \\
\text { control }\end{array}$ & 56.9098 \\
\hline $\mathrm{K}_{\mathrm{p} \omega}$ & $\begin{array}{l}\text { The proportional component for the turning } \\
\text { speed control }\end{array}$ & 2.05 \\
\hline $\mathrm{K}_{\mathrm{i} \omega}$ & $\begin{array}{l}\text { The integral component for the turning speed } \\
\text { control }\end{array}$ & 8.4803 \\
\hline
\end{tabular}

Table 1 Parameters of PD controllers for wheel torque control of the excavator base model given in [19] and [20].

The extended backstepping position controller which is proposed in paper [20] is used in the outer loop for position control. The position controller outputs are 
defined by (20) to (22) where the parameter values are given in Table 2.

\begin{tabular}{|l|l|}
\hline Parameter & Value \\
\hline $\mathrm{k}_{1}$ & 18.2620 \\
\hline $\mathrm{k}_{2}$ & 18.75 \\
\hline $\mathrm{k}_{3}$ & 9.8229 \\
\hline $\mathrm{k}_{4}$ & 26.5370 \\
\hline $\mathrm{k}_{5}$ & 1.0164 \\
\hline $\mathrm{k}_{6}$ & $\mathrm{k}_{6}=2.0028$ \\
\hline
\end{tabular}

Table 2 Parameters of the backstepping position control of the excavator base model given in [20].

$$
\begin{gathered}
{\left[\begin{array}{l}
e_{1} \\
e_{2} \\
e_{3}
\end{array}\right]=\left[\begin{array}{ccc}
\cos \theta_{c} & \sin \theta_{c} & 0 \\
-\sin \theta_{c} & \cos \theta_{c} & 0 \\
0 & 0 & 1
\end{array}\right]\left[\begin{array}{c}
x_{r}-x \\
y_{r}-y \\
\theta_{r}-\theta_{c}
\end{array}\right]} \\
u_{1}=v_{r} \cos e_{3}+\frac{k_{1} e_{1}}{\sqrt{k_{4}+e_{1}^{2}+e_{2}^{2}}} \\
u_{2}=\omega_{r}+\frac{k_{2} v_{r} e_{2}}{\sqrt{k_{5}+e_{1}^{2}+e_{2}^{2}}}+\frac{k_{3} v_{r} \sin e_{3}}{\sqrt{k_{6}+e_{3}^{2}}}
\end{gathered}
$$

\section{B. Robust control of the excavator arm}

Usually, the excavator is required to carry out tasks involving contact with its environment, such as levelling and digging. In moving towards autonomous excavation, it is necessary to develop a controller that is robust to uncertainties associated with such tasks.

Although there are some pronounced differences between the classical robot manipulator and robotic excavation, there are also some parallels. Therefore, there are many control approaches which have been developed for the robot manipulator that can be adopted by the robotic excavation. In this section, we will firstly study control of the excavator using the conventional computed torque control which has been developed on the fullyactuated robot manipulator. Then, we will develop a robust control approach which is effective to reject external disturbance during excavation. After that, extensive simulation results will be compared.

Using the dynamic model of the excavator arm in (5), the computed torque control (CTC) law is given as below:

$$
U_{a}=\hat{D}_{a}(\theta) \dot{\theta}_{v}+\hat{C}_{a}(\theta, \dot{\theta}) \dot{\theta}+\hat{G}_{a}(\theta)+\hat{B}_{a}(\dot{\theta})
$$

where $\dot{\theta}_{v}=\ddot{\theta}_{d}-k_{v} \dot{e}_{a}-k_{p} e_{a}, e_{a}=\theta-\theta_{d}, \mathrm{k}_{\mathrm{v}}$ and $\mathrm{k}_{\mathrm{p}}$ are linear gains to be designed, $\hat{D}_{a}(\theta)$ is the estimated inertia; $\hat{C}_{a}(\theta, \dot{\theta})$ is the estimated Coriolis and centripetal effects; $\hat{G}_{a}(\theta)$ is the estimated gravity forces; $\hat{B}_{a}(\dot{\theta})$ is the estimated friction effects, $\mathrm{U}_{\mathrm{a}}$ is the computed torques applied to the system, $\theta_{d}, \dot{\theta}_{d}, \ddot{\theta}_{d}$ are the desired joint link angle, angular velocity, and angular acceleration, respectively.

It is found that the CTC approach is specified by the inverse dynamics of the excavator (5). The controller (23) generates the generalized torques to be applied to the excavator producing the desired motion. The simulation is carried out in two cases: 1) tracking the desired motion without payload and 2) tracking the desired motion with payload $\left(\mathrm{M}_{\text {load }}=500 \mathrm{~kg}\right)$ but it is assumed to be unknown. The parameters of the excavator arm are given in Appendix. For both cases, the linear gains $\mathrm{k}_{\mathrm{v}}=100$ and $\mathrm{k}_{\mathrm{p}}=150$ are used.

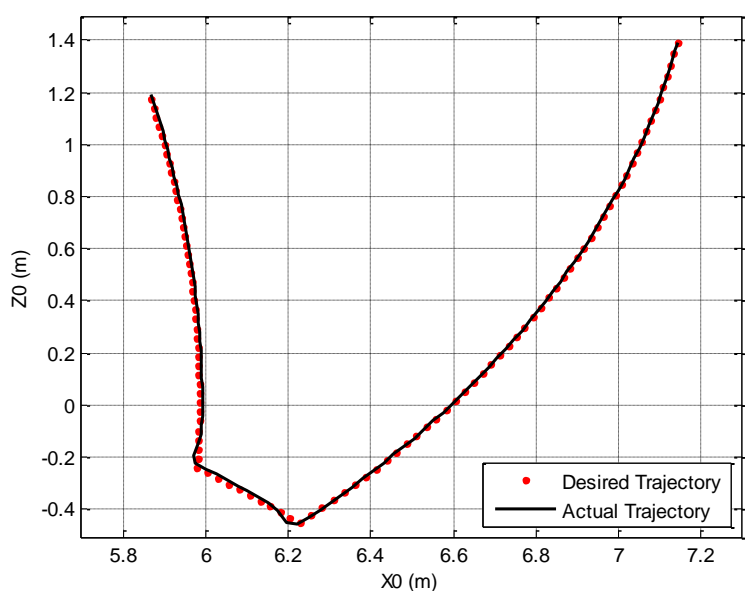

Figure 4 The bucket $\left(\mathrm{O}_{3}\right)$ trajectory under the CTC law without payload

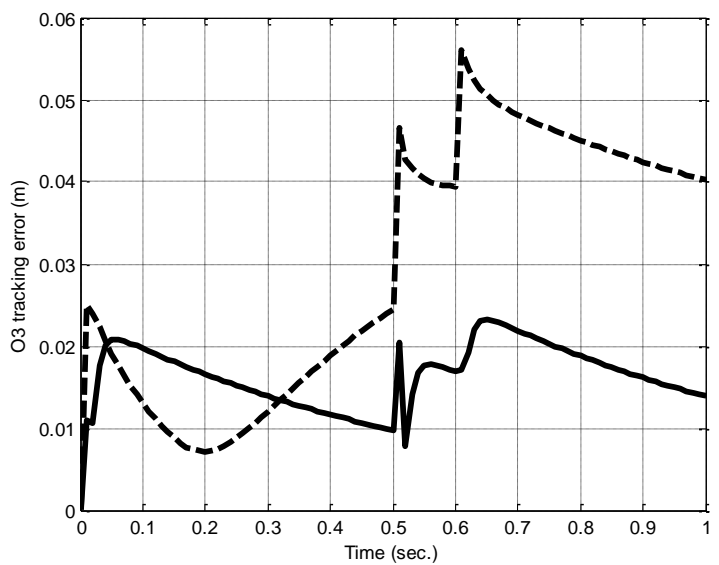

Figure 5 The bucket $\left(\mathrm{O}_{3}\right)$ tracking errors (solid line: without payload; dash line: with unknown payload)

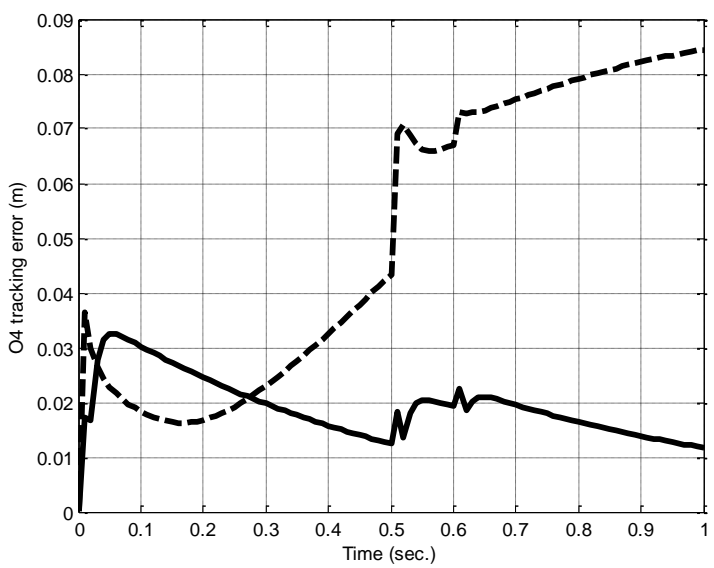

Figure 6 The bucket head $\left(\mathrm{O}_{4}\right)$ tracking errors (solid line: without payload; dash line: with unknown payload) 
The simulation results are presented as follows. In Figure 4, the actual bucket motion under the CTC law without payload is shown. To analyze the performance of the CTC law, Figure 5 and Figure 6 present the tracking errors of the bucket and the bucket head, respectively. The tracking error is the absolute distance from the actual trajectory to the desired trajectory. From Figure 5, it can be seen that the case with unknown payload gives a maximal tracking error which is about $0.056 \mathrm{~m}$, and the average tracking error with payload is apparently larger than the case without payload. Also, in Figure 6, the tracking performance is not as good as desired when the system is loaded with unknown payload. In Figure 7, the control inputs by using the CTC law with payload are given.
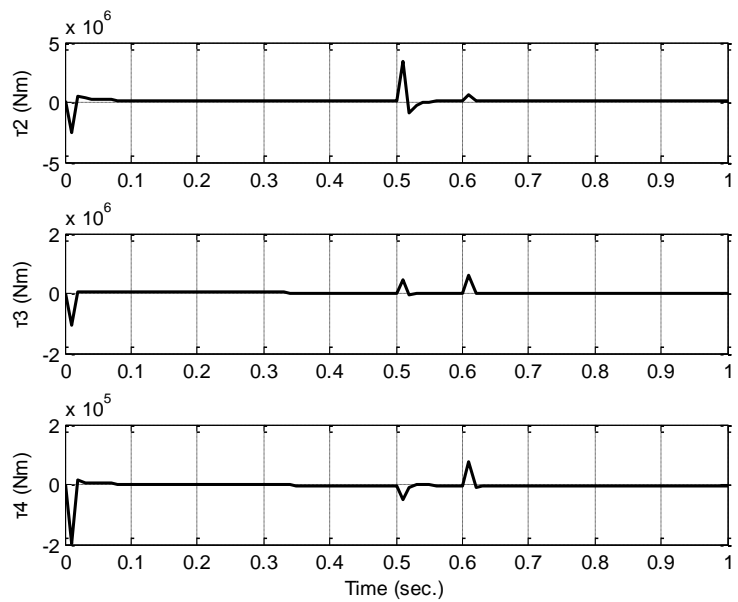

Figure 7 The control torques under the CTC law with unknown payload

From the simulation results above, it can be found that the CTC law cannot give a desired tracking performance under the case with unknown payload. So, a robust control approach is required to adapt to an uncertain circumstance. According to the dynamic model in (5), the robust control (RC) law [21] is introduced as below

$$
\begin{gathered}
\tilde{x}(t)=\left[\begin{array}{ll}
\dot{e}(t) & e(t)
\end{array}\right]^{T} \\
\bar{W}(t) \Theta(t)+\bar{W}_{0}(t) \\
=D(\theta)\left\{\dot{v}-\mu\left(\dot{e}+P_{12} e\right)\right\}+C(\theta, \dot{\theta}) v+G(\theta) \\
\mathrm{T}(\mathrm{t})=\mathrm{T}_{\mathrm{f}}(\mathrm{t})+\mathrm{T}_{1}(\mathrm{t}) \\
\mathrm{T}_{1}(\mathrm{t})=-\left(\mathrm{P}_{11}+\mathrm{P}_{\mathrm{cc}} \Gamma^{-1} \mathrm{P}_{\mathrm{cc}}\right) \mathrm{P}_{1} \tilde{x}(t)+P_{c c} e(t) \\
\mathrm{T}_{\mathrm{f}}(\mathrm{t})=\bar{W}(t) \Theta_{v}(t)+\bar{W}_{0}(t) \\
\Theta_{v}(t)=-F_{1}(t) \bar{\Theta}_{\theta_{i}} \geq \theta \forall i \\
F_{1}(t)=\operatorname{diag}\left\{\operatorname{sgn}\left(f_{1}\right), \operatorname{sgn}\left(f_{2}\right), \ldots, \operatorname{sgn}\left(f_{p}\right)\right\} \\
F(t)=\tilde{x}^{T} P_{1}^{T} \bar{W}(t)=\left[f_{1}(t), f_{2}(t), \ldots, f_{p}(t)\right]
\end{gathered}
$$

where $v=\dot{\theta}_{d}-P_{12} e, \Theta$ is a vector containing the unknown excavator and load parameters, which are known to lie in a bounded set: $\Omega=\left\{\Theta|| \theta_{i} \mid \leq \bar{\theta}_{i}, i=1,2, \ldots, p\right\}, \Theta_{\mathrm{v}}(\mathrm{t})$ is a switching-function vector, $\mathrm{P}_{c c}, \Gamma, \mathrm{P}_{11} \in \mathrm{R}^{\mathrm{nxn}}$ are symmetric positive definite matrices, $\mathrm{P}_{12}=\mathrm{P}_{\mathrm{cc}}{ }^{-1} \Gamma, \mathrm{P}_{1}=\left[\mathrm{I}_{\mathrm{nxn}} \mathrm{P}_{12}\right]$.

In the simulation, the coefficients are chosen as $\mathrm{P}_{\mathrm{cc}}=100 \mathrm{I}_{3 \times 3}, \mathrm{P}_{11}=80 \mathrm{I}_{3 \times 3}, \mathrm{P}_{12}=40 \mathrm{I}_{3 \times 3}$ and $\mu=3.5$. The resulting linear feedback control law is

$$
\mathrm{T}_{1}(\mathrm{t})=-82.5 \dot{e}(t)-3200 e(t)
$$

The payload in the bucket is assumed unknown, but with known bounds $0 \leq \mathrm{M}_{\text {load }} \leq \mathrm{M}_{\max }$, where $\mathrm{M}_{\max }=\mathrm{V}_{\mathrm{b}} \cdot \rho$ is the maximal weight that the excavator can load, $V_{b}$ is the volume of the bucket, $\rho$ is the soil density. In the simulation $\mathrm{M}_{\mathrm{load}}=500 \mathrm{~kg}$ and $\mathbf{M}_{\max }=1114.6 \mathrm{~kg}$. According to (28)-(31), the RC law is given as below:

$$
\begin{gathered}
\mathrm{f}(\mathrm{t})=[\{\dot{e}(t)+40 e(t)\} \bar{W}(t)] \\
\Theta_{v}(t)=-\left(M_{b u}+M_{\max }\right) \operatorname{sgn}\{f(t)\} \\
\mathrm{T}_{\mathrm{f}}(\mathrm{t})=\bar{W}(t) \Theta_{v}(t)+\bar{W}_{0}(t)
\end{gathered}
$$

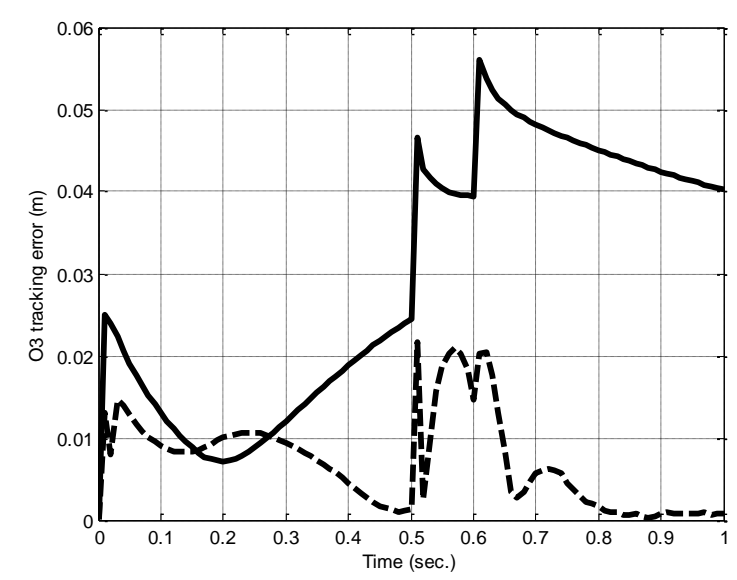

Figure 8 The bucket $\left(\mathrm{O}_{3}\right)$ tracking errors with unknown payload (solid line: the CTC law; dash line: the RC law)

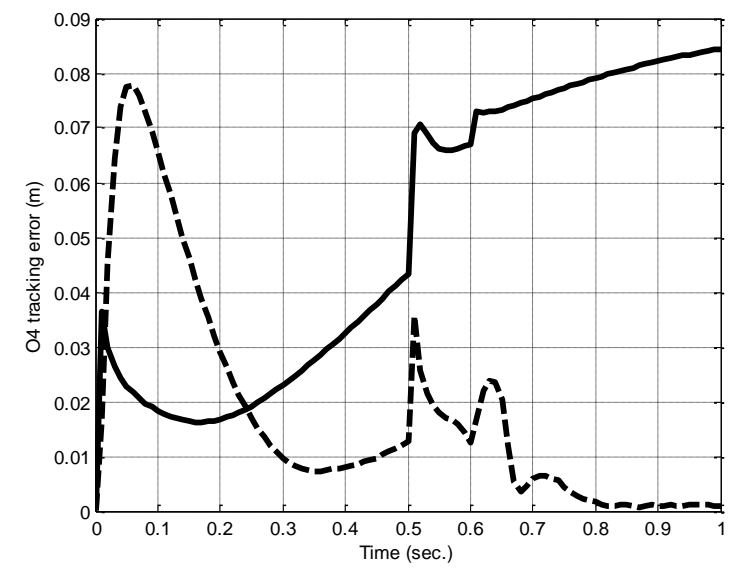

Figure 9 The bucket head $\left(\mathrm{O}_{4}\right)$ tracking errors with unknown payload (solid line: the CTC law; dash line: the RC law)

The comparison between the CTC law and the RC law is made by using the simulation results as follows. The tracking results of the desired bucket motion by using both control laws are presented in Figure 8. For both simulations, the mass of the payload is assumed unknown for the controller design. As shown in Figure 8, it can be 
seen that the CTC law gives a maximal tracking error which is about $0.056 \mathrm{~m}$, while the RC law gives a better tracking performance, i.e. the average tracking errors given by the RC law is obviously less than the average tracking errors given by the CTC law. In Figure 9, it can be found that the tracking results are consistent with the results shown in Figure 8. Although the RC law gives a large tracking error at the beginning, but the error becomes smaller finally. Therefore, the comparison can validate that the RC law is more effective and more robust to uncertain circumstance. Furthermore, the control torques by using the CTC law and the RC law are compared in Figure 10. From the figure, it can be found that the control inputs of the $\mathrm{RC}$ law are much larger than the control inputs of the CTC law. It gives a reasonable result that the RC law effectively reduces the tracking error during the excavation, and gives a better tracking performance.
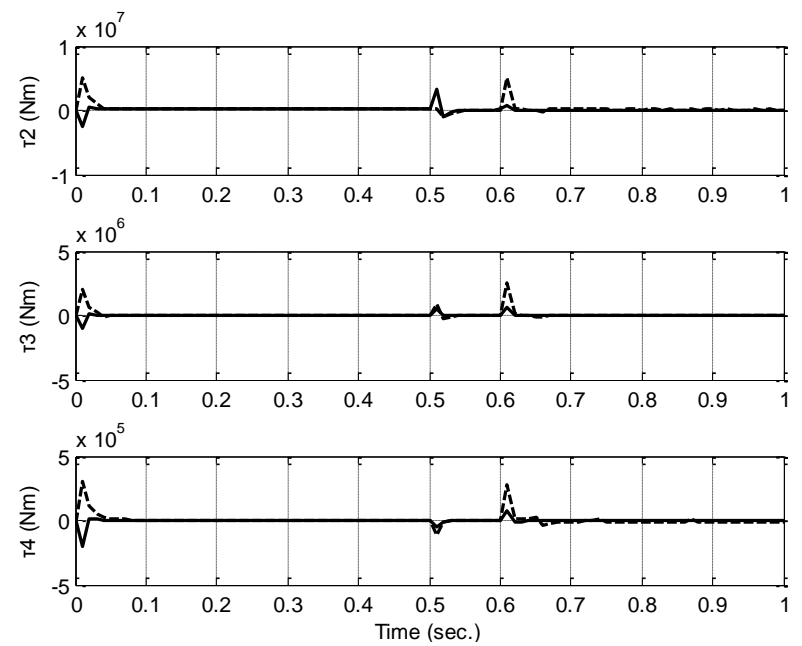

Figure 10 The control torques with unknown payload (solid line: the CTC law; dash line: the RC law)

\section{REMOTE CONTROL OF THE EXCAVATOR BASE}

The co-simulation framework [23][24] that utilizes MATLAB-SIMULINK to model the plant-controller and OPNET to simulate the network has been used to implement the position control of the excavator base and is shown in Figure 11. The co-simulation parameters are given in Table 3.

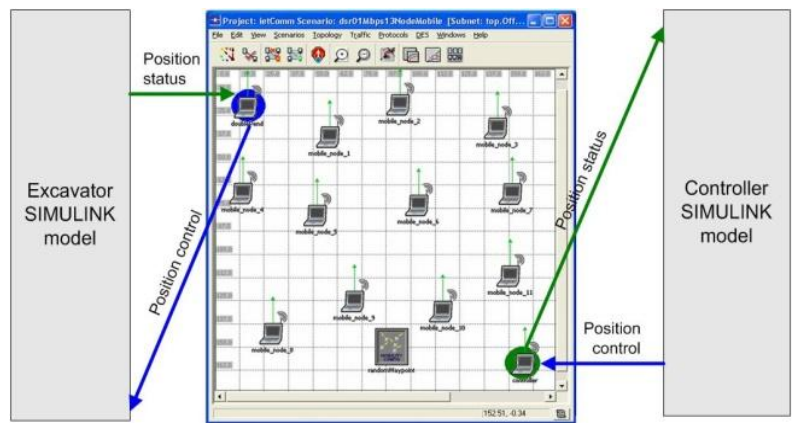

Figure 11 Interactive SIMULINK-OPNET co-simulation

The motive of position control is to control $\mathrm{x}$ and $\mathrm{y}$ coordinates as well as the orientation. The desired $\mathrm{x}$ and $\mathrm{y}$ coordinate profiles and the orientation or direction are taken from [19] and are shown in Figure 12(a), Figure 12(b) and Figure 12(c), respectively. The desired trajectory on $\mathrm{x}-\mathrm{y}$ plane with time of the excavator base is depicted in Figure 12(d).

\begin{tabular}{|c|c|}
\hline Parameter & Value \\
\hline Simulation area & 174 metre $\times 174$ metre \\
\hline Number of nodes & 13 \\
\hline $\begin{array}{l}\text { Wireless } \\
\text { communication } \\
\text { standard }\end{array}$ & The IEEE 802.11b (Direct Sequence) \\
\hline $\begin{array}{l}\text { Signal propagation } \\
\text { model }\end{array}$ & The pass loss and the fading [25][26] \\
\hline $\begin{array}{l}\text { MANET routing } \\
\text { protocol }\end{array}$ & The DSR protocol [27][28] \\
\hline MAC protocol & $\begin{array}{l}\text { The Carrier Sense Multiple Access with } \\
\text { Collision Avoidance (the CSMA/CA) [29] }\end{array}$ \\
\hline Packet size & 98 bytes \\
\hline Data rate & $11 \mathrm{Mbps}[30]$ \\
\hline Wireless card & $\begin{array}{l}\text { The Lucent ORINOCO wireless network } \\
\text { card [30] }\end{array}$ \\
\hline $\begin{array}{l}\text { Wireless card output } \\
\text { power }\end{array}$ & $15 \mathrm{dBm}[30]$ \\
\hline $\begin{array}{l}\text { Wireless card } \\
\text { reception sensitivity }\end{array}$ & $-82 \mathrm{dBm}(11 \mathrm{Mbps})[30]$ \\
\hline Connection protocol & $\begin{array}{l}\text { The User Datagram } \\
{[31][32][33][34]}\end{array}$ \\
\hline $\begin{array}{ll}\text { Node } & \text { movement } \\
\text { model } & \\
\end{array}$ & The random way-point model [35][36] \\
\hline Sampling mechanism & Clock driven [37][38][39][34][40] \\
\hline Control mechanism & $\begin{array}{l}\text { Event driven (upon the arrival of the state } \\
\text { packet) [37][38][39][34][40] }\end{array}$ \\
\hline Actuation mechanism & $\begin{array}{l}\text { Event driven (upon the arrival of the control } \\
\text { packet) [37][38][39][34][40] }\end{array}$ \\
\hline Desired output & $\begin{array}{l}\text { The reference } x, y \text { coordinates and } \\
\text { orientation with time, Figure } 12\end{array}$ \\
\hline Sampling period & $0.05 \mathrm{~s}[29][41][42]$ \\
\hline
\end{tabular}

Table 3 Parameters of SIMULINK-OPNET co-simulation for the excavator base position control given in [19] and [20].

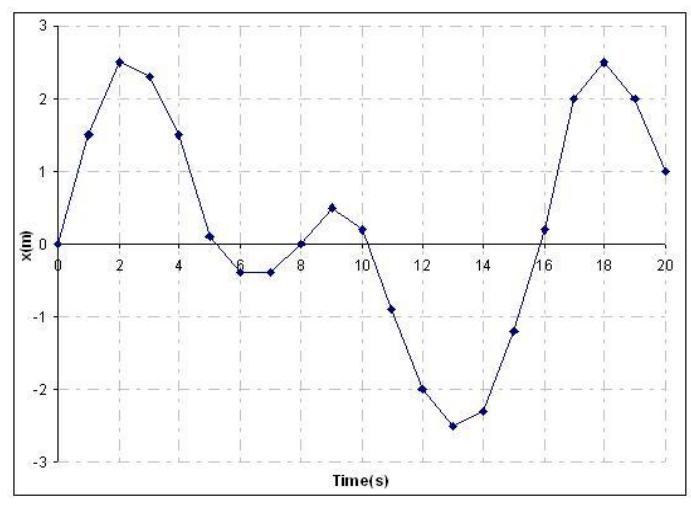

(a) Desired $x$ coordinates

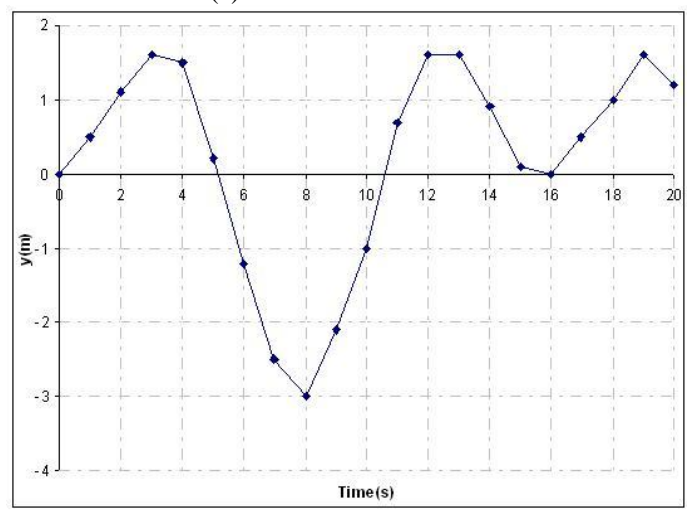

(b) Desired y coordinates 


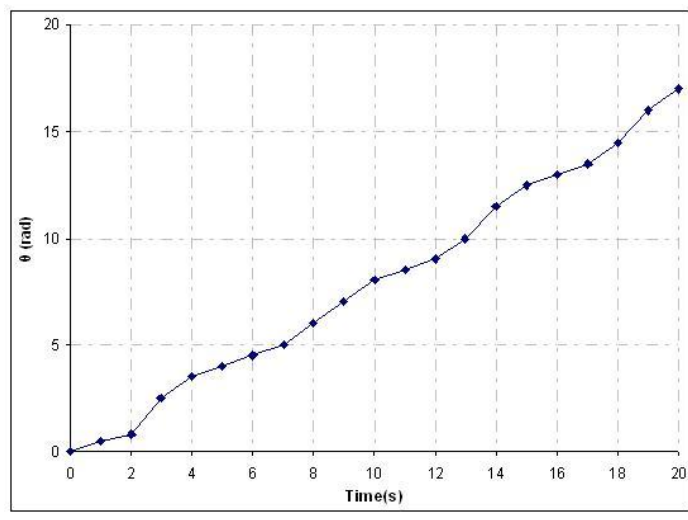

(c) Desired orientation

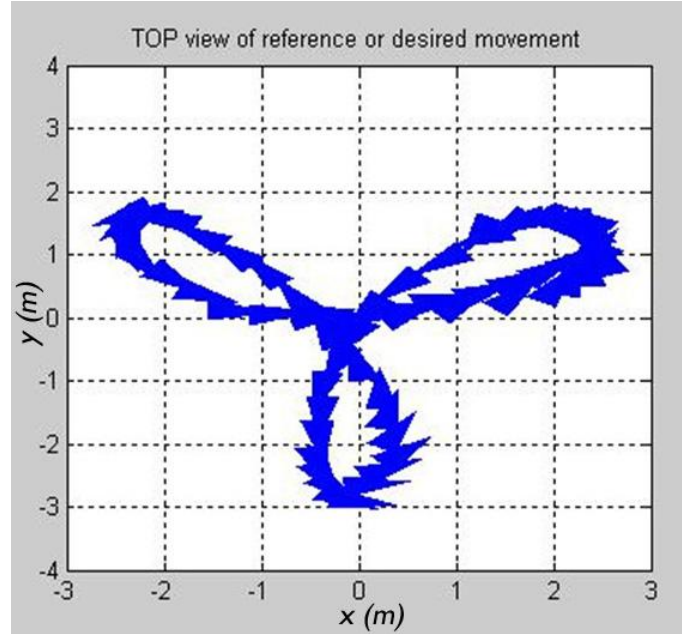

(d) Desired trajectory of the excavator base on $x$-y plane with time

Figure 12 The desired $\mathrm{x}, \mathrm{y}$ coordinates, orientation and trajectory with time [19].

The excavator model sends the $\mathrm{x}$ coordinate $x, \mathrm{y}$ coordinate $y$ and the orientation theta to the backstepping controller over the OPNET MANET model. The controller compares the $x, y$ and theta values with the reference $x \_r e f, y \_r e f$ and theta_ref and sends the required input $u_{1}$ and $u_{2}$ to the velocity controller at the excavator site. The trajectory of the excavator base at the data rate of $11 \mathrm{Mbps}$ is shown in Figure 13. The control torques for the excavator right and left wheels are shown in Figure 14 and Figure 15, respectively.

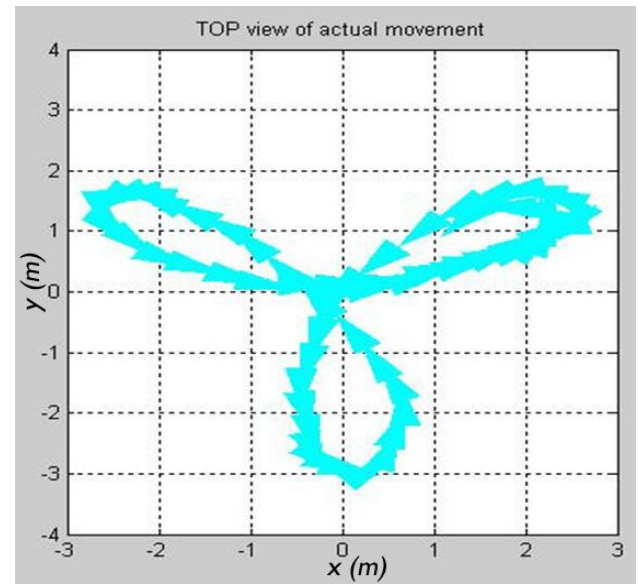

Figure 13 Actual trajectories of the excavator base under various data rates.

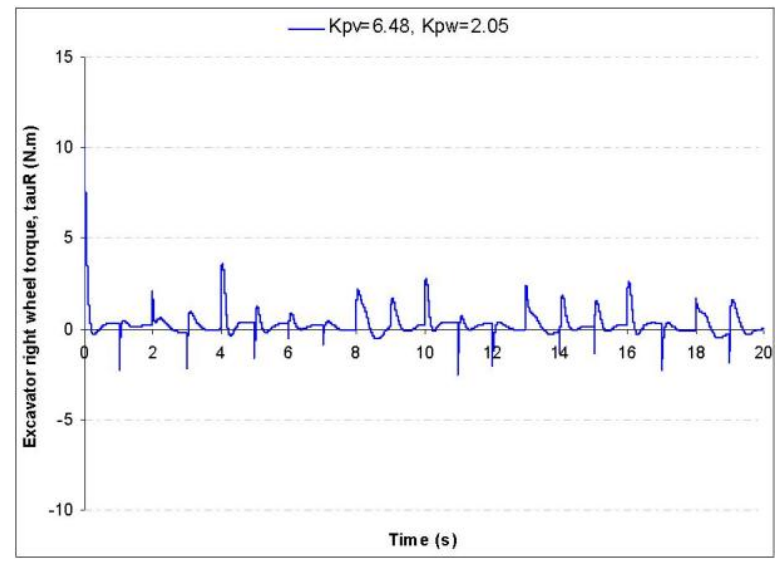

Figure 14 The control torque of the excavator right wheel

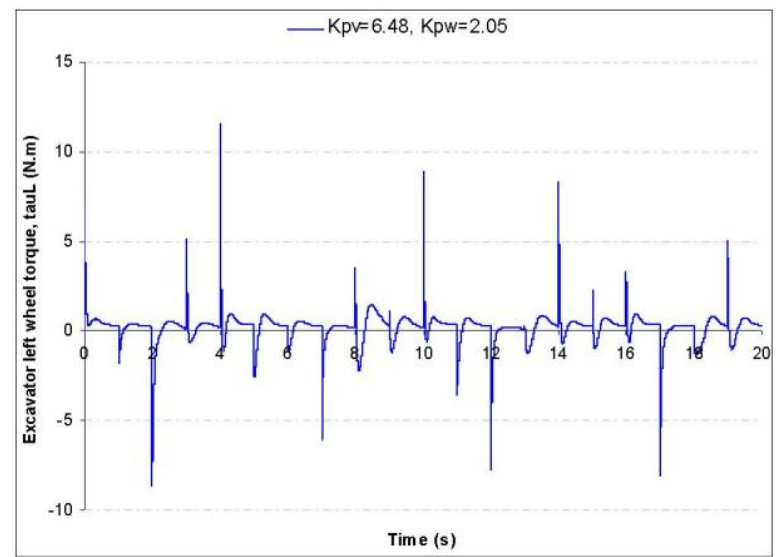

Figure 15 The control torque of the excavator left wheel

\section{CONCLUSIONS AND FURTURE WORK}

This paper has reported the work conducted in an ongoing project. The key issues in remotely controllable excavators have been identified. An overall architecture has been proposed and functions of each block of the architecture have been discussed. Some simulation work has been conducted to demonstrate the proposed system.

We will conduct further simulation on the whole system. The experimental study will also be investigated.

\section{ACKNOWLEDGEMENT}

The authors would like to thank Mr Sam Wane for the useful discussion and $\mathrm{J} \mathrm{C}$ Bamford Excavators Limited, Sellafield Limited for initiating this work.

\section{APPENDIX}

Parameters of the excavator base model:

$\mathrm{M}=10 \mathrm{~kg}$ : mass of the entire base

$\mathrm{I}_{\mathrm{A}}=1.0 \mathrm{~kg} \cdot \mathrm{m}^{2}$ : moment of inertia of the entire base considering point $\mathrm{A}$

$\mathrm{I}_{0}=0.001 \mathrm{~kg} \cdot \mathrm{m}^{2}:$ moment of inertia of the wheel complex

$\mathrm{L}=0.35 \mathrm{~m}$ : width of the base

$\mathrm{r}=0.035 \mathrm{~m}$ : radius of the wheels

$\mathrm{d}=0.05 \mathrm{~m}$ : distance between point $\mathrm{A}$ and $\mathrm{C}$

$\theta_{c}$ : angle representing the orientation of the base

$\theta_{\mathrm{R}}$ : angle position of the right wheel

$\theta_{\mathrm{L}}$ : angle position of the left wheel

$\tau_{\mathrm{R}}$ : actuation torque of the right wheel 
$\tau_{\mathrm{L}}:$ actuation torque of the left wheel

Parameters of the excavator arm model:

$\mathrm{M}_{\mathrm{bo}}=1566 \mathrm{~kg}$ : mass of boom

$\mathrm{M}_{\mathrm{st}}=735 \mathrm{~kg}$ : mass of stick

$\mathrm{M}_{\mathrm{bu}}=432 \mathrm{~kg}$ : mass of bucket

$\mathrm{M}_{\text {load }}=500 \mathrm{~kg}$ : mass of load

$\mathrm{V}_{\mathrm{b}}=0.58 \mathrm{~m}^{3}$ : volume of bucket

$\rho=1921.8 \mathrm{~kg} / \mathrm{m}^{3}$ : soil density

$\mathrm{M}_{\max }=\mathrm{V}_{\mathrm{b}} \cdot \rho$ : maximal load weight

$\mathrm{I}_{\mathrm{bo}}=14250.6 \mathrm{~kg} \cdot \mathrm{m}^{2}$ : moment of inertia of boom

$\mathrm{I}_{\mathrm{st}}=727.7 \mathrm{~kg} \cdot \mathrm{m}^{2}:$ moment of inertia of stick

$\mathrm{I}_{\mathrm{bu}}=224.6 \mathrm{~kg} \cdot \mathrm{m}^{2}:$ moment of inertia of bucket

$\theta_{1}$ : angle of base

$\theta_{2}$ : angle of boom

$\theta_{3}$ : angle of stick

$\theta_{4}$ : angle of bucket

$\theta_{\mathrm{b}}$ : angle between bucket bottom and $\mathrm{X}_{4}$-axis

$\theta_{\mathrm{dg}}$ : angle between bucket edge and horizontal line

$\mathrm{a}_{1}=0.05 \mathrm{~m}: \mathrm{O}_{0} \mathrm{O}_{1}$

$\mathrm{a}_{2}=5.16 \mathrm{m:} \mathrm{O}_{1} \mathrm{O}_{2}$

$\mathrm{a}_{3}=2.59 \mathrm{m:} \mathrm{O}_{2} \mathrm{O}_{3}$

$\mathrm{a}_{4}=1.33 \mathrm{m:} \mathrm{O}_{3} \mathrm{O}_{4}$

$\mathrm{r}_{2}=2.71 \mathrm{m:} \mathrm{O}_{1} \mathrm{G}_{2}$

$\mathrm{r}_{3}=0.64 \mathrm{m:} \mathrm{O}_{2} \mathrm{G}_{3}$

$\mathrm{r}_{4}=0.65 \mathrm{m:} \mathrm{O}_{3} \mathrm{G}_{4}$

$\alpha_{2}=0.2566 \mathrm{rad}: \angle \mathrm{G}_{2} \mathrm{O}_{1} \mathrm{O}_{2}$

$\alpha_{3}=0.3316 \mathrm{rad}: \angle \mathrm{G}_{3} \mathrm{O}_{2} \mathrm{O}_{3}$

$\alpha_{4}=0.3944 \mathrm{rad}: \angle \mathrm{G}_{4} \mathrm{O}_{3} \mathrm{O}_{4}$

$\mathrm{B}_{\mathrm{bo}}$ : viscous friction coefficient of boom

$B_{s t}:$ viscous friction coefficient of stick

$\mathrm{B}_{\mathrm{bu}}$ : viscous friction coefficient of bucket

$\mathrm{g}=9.81 \mathrm{~N} / \mathrm{kg}$ : acceleration due to gravity

$\mathrm{T}_{\mathrm{s}}=10 \mathrm{~ms}$ : sampling time

\section{REFERENCES}

[1] H. Yu, Y. Liu, and M. S. Hasan, "Review of modelling and remote control for excavators," International Journal of Advanced Mechatronic Systems (accepted for publication), vol. 2, no. 1/2, 2009.

[2] A. J. Koivo, "Kinematics of excavators (backhoes) for transferring surface material," J. Aerosp. Eng, vol. 7, no. 1, pp. 17-32, 1994.

[3] P. K. Vaha and M. J. Skibniewski, "Dynamic model of excavator," J. Aerosp. Eng., vol. 6, pp. 148-158, 1993.

[4] A. J. Koivo, M. Thoma, E. Kocaoglan, and J. AndradeCetto, "Modelling and control of excavator dynamics during digging operation," J. Aerosp. Eng., vol. 9, no. 1, pp. 10-18, 1996.

[5] S. Tafazoli, P. D. Lawrence, and S. E. Salcudean, "Identification of inertial and friction parameters for excavator arms," IEEE Trans. on Robotics and Automation, vol. 15, no. 5, pp. 966-971, 1999.

[6] Y. H. Zweiri, "Identification Schemes for Unmanned Excavator Arm Parameters," Int. J. Automation and Computing, vol. 05, no. 2, pp. 185-192, 2008.

[7] C. P. Tan, Y. H. Zweiri, K. Althoefer, and L. D. Seneviratne, "Online soil parameter estimation scheme based on Newton-raphson method for autonomous excavation," IEEE/ASME Trans. on Mechatronics, vol. 10, no. 2, pp. 221-229, 2005.

[8] S. Tafazoli, Salcudean, S. E., Hashtrudi-Zaad, K., and Lawrence, P. D., "Impedance control of a teleoperated excavator," IEEE Trans. on Control Systems Technology, vol. 10, no. 3, pp. 355-367, 2002.

[9] Z. Lu and A. A. Goldenberg, "Robust impedance control and force regulation: theory and experiment," Int. J. Robotic. Res., vol. 14, no. 3, pp. 225-254, 1995.

[10] Q. P. Ha, Q. H. Nguyen, D. C. Rye, and H. F. DurrantWhyte, "Impedance control of a hydraulically-actuated robotic excavator," Automat. Construction, vol. 9, no. 5, pp. 421-435, 2000.

[11] L. E. Bernold, "Motion and path control for robotic excavation," J. Aerosp. Eng., vol. 6, no. 1, pp. 1-18, 1993.

[12] S. Singh, "Synthesis of tactical plans for robotic excavation," in Robot. Inst., PhD thesis, Carnegie Mellon University, Pittsburgh, PA, USA, 1995.

[13] P. Saeedi, P. D. Lawrence, D. G. Lowe, J. P., D. Kusalovic, K. Ardron, and P. H. Sorensen, "An autonomous excavator with vision-based track-slippage control," IEEE Trans. on Control Systems Technology, vol. 13, no. 1, pp. 67-84, 2005.

[14] N. R. Parker, S. E. Salcudean, and P. D. Lawrence, "Application of force feedback to heavy duty hydraulic machines," presented at the IEEE Conf. on Robotics and Automation, Atlanta, USA, 1993.

[15] D. Kim, K. W. Oh, D. Hong, J. Park, and S. Hong, "Remote control of excavator with designed haptic device," presented at the Int. Conf. on Control, Automation and Systems, Seoul, Korea, 2008.

[16] S. P. Dimaio, S. E. Salcudean, C. Reboulet, S. Tafazoli, and K. H. Zaad, "A virtual excavator for controller development and evaluation," presented at the IEEE Int. Conf. on Robotics and Automation, 1998.

[17] N. Tao, D. Zhao, H. Yamada, and N. Shui, "A low-cost solution for excavator simulation with realistic visual effect," presented at the IEEE Conf. on Robotics, Automation and Mechatronics, 2008.

[18] Y. Sakaida, D. Chugo, H. Yamamoto, and H. Asama, "The analysis of excavator operation by skilful operator," presented at the SICE Annual Conf., Japan, 2008.

[19] J. Velagic, B. Lacevic, and N. Osmic, "Nonlinear Motion Control of Mobile Robot Dynamic Model," in Mobile Robots Motion Planning, New Challenges. Vienna: Advanced Robotic System International, 2008, pp. 531552.

[20] B. Lacevic, J. Velagic, and B. Perunicic, "Reduction of Control Torques of Mobile Robot Using Hybrid Nonlinear Position Controller," presented at the International Conference on Computer as a Tool, EUROCON 2005, Serbia \& Montenegro, Belgrade, pp. 314-317, 2005.

[21] H. Yu, "Robust combined adaptive and variable structure adaptive control of robot manipulators," Robotica, vol. 16, pp. 623-650, 1998.

[22] T. V. Alekseeva, K. A. Artem'ev, A. A. Bromberg, R. L. Voitsekhovskii, and N. A. Ul'yanov, Machines for Earthmoving Work: Theory and Calculations: Rotterdam : A.A. Balkema, 1986.

[23] M. S. Hasan, H. Yu, A. Carrington, and T. C. Yang, "Cosimulation of wireless networked control systems over mobile ad hoc network using SIMULINK and OPNET," the Journal of IET Communications, vol. 3, no. 8, pp. 1297-1310, 2009.

[24] M. S. Hasan, H. Yu, A. Griffiths, and T. C. Yang, "Interactive co-simulation of MATLAB and OPNET for Networked Control Systems," presented at the 13th International Conference on Automation and Computing, Stafford, UK, pp. 237-242, 2007.

[25] K. Fall and K. Varadhan, "The Ns Manual (formerly ns Notes and Documentation), The VINT project," 2006, 
Available: http://www.isi.edu/nsnam/ns/doc/ns_doc.pdf, Mar 2006.

[26] S. Han and N. B. Abu-Ghazaleh, "On the Effect of Fading on Ad-hoc Networks," 2005, Available: http://arxiv.org/PS_cache/cs/pdf/0504/0504002v1.pdf.

[27] C. E. Perkins, Ad Hoc Networking. Boston, USA: AddisonWesley, 2001

[28] D. B. Johnson, D. A. Maltz, and J. Broch, Ad Hoc Networking. Boston, USA: Addison-Wesley, 2001.

[29] F. D. Pellegrini, D. Miorandi, S. Vitturi, and A. Zanella, "On the Use of Wireless Networks at Low Level of Factory Automation Systems," IEEE Transactions on Industrial Informatics, vol. 2, no. 2, pp. 129-143, 2006.

[30] Lucent Technologies Inc., "ORiNOCO PC Card, Home, office, and Public Mobile Broadband Internet Access," 2000 ,

Available: http://www.a1datacom.com/pdf/wavelan_PC.pdf, Jul 2009.

[31] M. Conti and S. Giordano, "Multihop Ad Hoc Networking: The Theory," IEEE Communications Magazine, vol. 45, no. 4, pp. 76-86, 2007.

[32] K. Goldberg, S. Gentner, C. Sutter, and J. Wiegley, "The Mercury Project: A Feasibility Study for Internet Robots," the IEEE Robotics and Automation Magazine, vol. 7, pp. 35-40, 2000.

[33] P. X. Liu, M. Meng, X. Ye, and J. Gu, "An UDP-Based Protocol for Internet Robots," presented at the 4th World Congress on Intelligent Control and Automation, China, vol. 1, pp. 59-65, 2002.

[34] N. J. Ploplys, P. A. Kawka, and A. G. Alleyne, "Closedloop Control over Wireless Networks," IEEE Control Systems Magazine, vol. 24, no. 3, pp. 58-71, 2004.

[35] J. Liu, Y. Yuan, D. M. Nicol, R. S. Gray, C. C. Newport, D. F. Kotz, and L. F. Perrone, "Simulation validation using direct execution of wireless ad-hoc routing protocols," presented at the 18th Workshop on Parallel and Distributed Simulation (PADS'04), Austria, pp. 7-16, 2004.
[36] C. Newport, "Simulating mobile ad hoc networks: a quantitative evaluation of common MANET simulation models," Dartmouth College Science Technical Report TR2004-504, 2004, Available: http://cmc.cs.dartmouth.edu/cmc/papers/newport:thesis.pdf

[37] W. Zhang, M. S. Branicky, and S. M. Phillips, "Stability of Networked Control Systems," IEEE Control Systems Magazine, vol. 21, no. 1, pp. 84-99, 2001.

[38] J. Colandairaj, G. W. Irwin, and W. G. Scanlon, "An integrated approach to wireless feedback control," presented at the UKACC International Control Conference, Glasgow, UK, 2006.

[39] N. J. Ploplys, "Wireless feedback control of mechanical systems," in Mechanical Engineering, MSc thesis, University of Illinois, Champaign, 2003, Available: http://mrroboto.me.uiuc.edu/ARGWeb/theses/NicksThesis/Ploplys MS2003.pdf.

[40] S. Zampieri, "Trends in Networked Control Systems," presented at the 17th World Congress, the International Federation of Automatic Control, Seoul, Korea, pp. 28862894, 2008.

[41] J. Nilsson, "Real-time Control Systems with Delays," in Automatic Control, $\mathrm{PhD}$ thesis, Lund Institute of Technology, Lund, Sweden, 1998, Available: http://www.control.lth.se/documents/1998/nilj98dis.pdf.

[42] D. Henriksson, "Flexible Scheduling Methods and Tools for Real Time Control Systems," in Automatic Control, $\mathrm{PhD}$ thesis, Lund Institute of Technology, Lund, Sweden, 2003, Available: http://www.control.lth.se/documents/2003/hen03.pdf. 